\title{
Meta-Analysis of Atrial Electro-Gram Applied to PICC Catheter Tip Positioning
}

\author{
Jian Song1, ChunLin Wang ${ }^{2}$, Jun-E Hu \\ ${ }^{1}$ Department of Medical College, Yangtze University, Jingzhou, China \\ ${ }^{2}$ Department of oncology, Jing Zhou Central Hospital, Jingzhou, China \\ Email:654546433@qq.com
}

How to cite this paper: Song, J., Wang, C.L. and Hu, J.-E. (2017) Meta-Analysis of Atrial Electro-Gram Applied to PICC Catheter Tip Positioning. Yangtze Medicine, 1, 65-76.

https://doi.org/10.4236/ym.2017.12007

Received: March 9, 2017

Accepted: June 18, 2017

Published: June 21, 2017

Copyright (c) 2017 by authors and Scientific Research Publishing Inc. This work is licensed under the Creative Commons Attribution International License (CC BY 4.0).

http://creativecommons.org/licenses/by/4.0/

\begin{abstract}
Objective: Assess the location effect of the atrial electrogram in the PICC catheter tip. Method: Make online research in the CNKI, CBM, Wanfang, VIP, Database, Cochrane Library, JBI Library, PubMed, Ovid, Springer, Web of science, MEDLINE database to collect the randomized controlled trial data of using the atrial electrogram to locate the PICC catheter tip. When making the online research, only the data to November 20, 2016 can be searched out. Two researchers independently select the literature and information, make quality assessment, and then use the RevMan 5.2 software to make Meta analysis. Result: 16 random tests are done. The Meta analysis results show that compared with the traditional $\mathrm{X}$ ray chest radiograph location method, the atrial electrogram can increase the accuracy rate of the location of the catheter tip $[\mathrm{RR}=$ $1.13,95 \% \mathrm{CI}(1.07,1.19), \mathrm{P}<0.00001]$; it can decrease the incidence rate of related complications $[\mathrm{RR}=0.37,95 \% \mathrm{CI}(0.21,0.66), \mathrm{P}<0.0008]$; and it can save the catheter placing time $[\mathrm{RR}=-12.56,95 \% \mathrm{CI}(-18.56,-6.57), \mathrm{P}<0.0001]$. Conclusion: The atrial electrogram can increase the accuracy rate of the location of the catheter tip, decrease the incidence rate of related complications and save the catheter placing time. Therefore, it is worthy of clinical application.
\end{abstract}

\section{Keywords}

Atrial Electrogram, PICC Catheter, Meta

\section{Introduction}

Peripherally inserted central catheter (PICC) has been widely applied in our country due to its advantages, namely, long-term use, reduced puncture pain, safety, efficiency, resistance to hypertonic and intense irritant drugs as well as fewer complications [1]. In order to achieve the effect of peripheral venous 
puncture and central venous therapy, the position of PICC tip is essential. American Society for Intravenous Infusion (INS) recommended PICC tip should be located at the lower $1 / 3$ of patient's superior vena cava [2]. At present, X-ray film combined with in vitro measurement method is adopted for the location of PICC tip in domestic. With the progress of the discipline, X-ray positioning method, as the "gold standard" of PICC tip location shows its hysteretic nature [3], and it is gradually replaced by atrial electro-gram due to its drawback of radiation damage for pregnant women, children and other special groups. In clinical work, there are many studies regarding the application of atrial electro-gram on PICC catheter tip location, but the majority of them are lack of evidences and clinical practice guidelines to confirm the accuracy and superiority [4] [5]. The aim of this study is to investigate the accuracy and advantages of atrial electro-gram in the location of the tip of PICC catheters, with a view to providing references for clinical care and the clinical practice guidelines of selecting a tip location method after PICC catheterization.

\section{Materials and Methods}

\subsection{Document Inclusion and Exclusion Criteria (PICOD Principles)}

\subsubsection{Study Subject (Population)}

1) PICC catheterized patients aged over 18 and voluntary to participate in this research. 2) Patients with body surface electrocardiograms being normal before catheterizing. 3) Patients whose catheter head ends were localized through intra-atrial electrocardiogram technology. 4) Patients who didn't have heart diseases or previous operation history of heart diseases. Exclusion criterion: 1) Patients whose electrocardiographic examination results were normal before catheterizing such as patients with atrial fibrillation and ventricular tachycardia and those carrying cardiac pacemakers.

\subsubsection{Intervening Measure (Intervention)}

In the control group: The PICC catheter was implanted with the predicted length according to the length of the in vitro measurement. X-ray examination was performed to determine that the tip of the catheter was located in the lower $1 / 3$ of the superior vena cava.

\subsubsection{Control Measure (Control)}

Pick up the surface ECG, observe the ECG P wave changes during the catheterization process in real time, and then determine the catheter tip should be in the lower $1 / 3$ of the superior vena cava based on the specificities of $P$ wave, further $\mathrm{X}$-ray examination should be performed to verify the location of the tip of PICC cathether [6].

\subsubsection{Outcome Indicator (Outcome)}

The main outcome indicator is estimation accuracy of intra-atrial electrocardiogram technology for PICC catheter head end to reach ideal position. Secondary 
indicators mainly include occurrence rate catheter-related complications and the time taken by catheterizing.

\subsubsection{Study Design (Design)}

Randomized Controlled Trial (RCT) of atrial electro-gram applied to PICC catheter head-head location. Exclusion criteria: non-randomized controlled trials, clinical controlled trials, systematic review.

\subsection{Literature Retrieval Strategy}

Using "Peripherally inserted central catheter", "PICC", "Catheter tip location", "Atrial electro-gram positioning”, "Electrocardiography", "ECG”, "EKG” as key words ,the authors searched the CNKI, CBM, WanFang, VIP databases, Cochrane Library, JBI Library, PubMed, Ovid, Springer, Web of science, and MEDLINE databases. The time limit of these databases is from the establishment to April, 2016, and the retrieval is conducted in the form of free words with a keyword. In this study, CNKI and PubMed are taken as an example to introduce specific retrieval strategy [7] [8].

\subsection{Literature Screening and Data Extraction}

Two researchers independently screened the literature according to the inclusion and exclusion criteria set out in this study. By reading the literature title and abstract, the literature that clearly did not conform to the study was excluded. The controversial literature shall be discussed to decide whether or not to be included. If the result of the discussion is not consistent, a third party shall be asked to decide. For the literatures that meet the inclusion criteria, the information needed for this study, including the author's name, the time of publication, the source of the literature, the baseline data comparability, the sample size, as well as the intervening measures and outcome indicators of intervention group and the control group, were included in the study.

\subsection{Document Quality Evaluation}

The literature quality for randomized controlled trials and semi-randomized controlled trials is evaluated according to the quality criteria of Cochrane Review's Handbook [9]. The evaluation criteria specifically include: 1) whether the random order is generated correctly; 2) whether the allocation of random programs is hidden; 3 ) whether the blind method is used; 4) reports about the withdrawal and loss to follow-up; 5) whether the outcome indicators are complete; 6) baseline data comparability. The quality of the literature is divided into A, B, C three grades; Grade A fully meets the above criteria, Grade A meets part of the above criteria; and Grade $\mathrm{C}$ does not meet any of the above criteria.

\subsection{Statistical Methods}

Meta-analysis was performed using Rev Man 5.2 software. The outcome index was classified as binary information, and used the RR (relative risk) value or OR 
(odds risk) as effective size. The continuous variable was expressed by using the weighted mean difference MD as the effect size, and the first thing was to determine whether there is heterogeneity in the study. If there is no heterogeneity in different studies $\left(\mathrm{P}>0.1, \mathrm{I}^{2}<50 \%\right)$ the fixed effect model is selected; if heterogeneity exists in different studies. $\left(\mathrm{P}<0.1, \mathrm{I}^{2}>50 \%\right)$, then it is necessary to analyze the reason for the heterogeneity. If there is statistical heterogeneity but no clinical heterogeneity among the various studies, random effects model will be adopted [7]; if the source of heterogeneity can't be determined, descriptive analysis will be adopted.

\section{Results}

\subsection{Literature Retrieval Results}

576 articles were retrieved in the relevant databases, after reading the title and abstract, 531 articles of repeated publication or obviously do not meet the inclusion criteria were excluded, so 45 articles were included initially. In the following, 29 articles with unclear interventions, no outcome evaluation indicators or low quality were also excluded after reading the full text, and 16 articles were finally included [10]-[25]. Meta-analysis was performed on 16 articles incorporating qualitative synthesis.

\subsection{General Condition of Included Literature}

A total of 16 randomized controlled trials were included. The total sample size was 2996. In the experimental group, atrial electro-gram real time location was used as an intervention measure during the catheterization, and then X-ray examination was used to confirm the accuracy of atrial electro-gram positioning; In the control group, $\mathrm{X}$-ray examination was used as an intervention measure to have the catheter tip location after the end of the catheterization. The observed indicators were catheter tip arrival rate, complication rate, catheterization timeconsuming. The general situation of the study literature is shown in Table 1.

\subsection{Evaluation of Literature Quality}

The 16 studies included were evaluated according to the Cochrane Review's Handbook quality assessment criteria, among the 16 studies that included in Meta-analysis, 10 studies [11] [12] [14] [15] [16] [18] [19] [21] [22] [25] specifically described the grouping method, and six studies [10] [13] [17] [20] [23] [24] reported only the using of random allocation, but not specifically described the grouping method; 3 studies [10] [12] [14] used single-blind, 1 study [14] used the envelope drawing method to follow the allocation of concealment; 5 studies [11] [12] [13] [14] [24] reported in detail the number and reasons of withdrawal of the sample; 1 study [14] conducted ITI analysis; the baseline information of 16 studies was comparable; the quality of 1 literature was Grade A, and the quality of the following literature [10] [11] [12] [13] [15]-[25] was Grade B. The quality evaluation included in the study was shown in Table 2 . 
Table 1. General condition of the 16 literatures included.

\begin{tabular}{|c|c|c|c|c|c|}
\hline \multirow{2}{*}{ Included research } & \multicolumn{2}{|c|}{ Sample size } & \multicolumn{2}{|c|}{ Intervention measure } & \multirow{2}{*}{$\begin{array}{l}\text { Outcome } \\
\text { indicator }\end{array}$} \\
\hline & $\begin{array}{l}\text { Experimental } \\
\text { group }\end{array}$ & $\begin{array}{l}\text { Control } \\
\text { group }\end{array}$ & $\begin{array}{l}\text { Experimental } \\
\text { group }\end{array}$ & $\begin{array}{l}\text { Control } \\
\text { group }\end{array}$ & \\
\hline Liu et al. [10] 2015 & 85 & 85 & I & II & $\mathrm{a}$ \\
\hline Elli et al. [11] 2016 & 77 & 44 & I & II & a \\
\hline Baldinelli et al. [12] 2015 & 42 & 48 & I & II & a \\
\hline Lei Wenlan et al. [13] 2016 & 130 & 130 & I & II & a \\
\hline Pan Longfang et al. [14] 2015 & 90 & 90 & I & II & $a, b, c$ \\
\hline Zhu Ya et al. [15] 2010 & 65 & 64 & I & II & a \\
\hline Lin Cuifang et al. [16] 2015 & 60 & 60 & I & II & $\mathrm{a}, \mathrm{b}$ \\
\hline Zhang Ronghua et al. [17] 2015 & 45 & 45 & I & II & $\mathrm{a}$ \\
\hline Zhou Lianqing et al. [18] 2013 & 51 & 49 & I & II & a, c \\
\hline Zhuo Yajuan et al. [19] 2014 & 60 & 60 & I & II & $\mathrm{a}, \mathrm{b}$ \\
\hline Xu Mingming et al. [20] 2012 & 70 & 70 & I & II & $a, b$ \\
\hline Jiang Guichun et al. [21] 2016 & 500 & 500 & I & II & a \\
\hline Wang Chaohong et al. [22] 2016 & 43 & 40 & I & II & $\mathrm{a}, \mathrm{b}$ \\
\hline Li Xiangjun et al. [23] 2016 & 98 & 106 & I & II & a \\
\hline Zheng Chunhua et al. [24] 2016 & 60 & 60 & I & II & a \\
\hline Ren Xiaomin et al. [25] 2016 & 35 & 35 & I & II & a, c \\
\hline
\end{tabular}

Note: 1) indicates the verified catheter tip position after the atrial electro-gram and X-ray chest positioning: indicates the verified catheter tip position after traditional catheterization and X-ray chest positioning. 2) a indicates the Arrival rate of catheter tip, $\mathrm{b}$ indicates the complication rate, $\mathrm{c}$ indicates the catheterization time-consuming.

Table 2. Quality evaluation results of 16 literatures included.

\begin{tabular}{|c|c|c|c|c|c|c|c|}
\hline Included research & Random method & $\begin{array}{l}\text { Blind } \\
\text { method }\end{array}$ & $\begin{array}{l}\text { Allocation } \\
\text { concealment }\end{array}$ & $\begin{array}{l}\text { Withdrawal and } \\
\text { loss to follow-up }\end{array}$ & $\begin{array}{c}\text { ITI } \\
\text { analysis }\end{array}$ & $\begin{array}{c}\text { Baseline } \\
\text { comparability }\end{array}$ & $\begin{array}{l}\text { Quality } \\
\text { grade }\end{array}$ \\
\hline Liu et al. [10] 2015 & unclear & single-blind & unclear & No & No & Yes & B \\
\hline Elli et al. [11] 2016 & random number & unclear & unclear & Yes & No & Yes & B \\
\hline Baldinelli et al. [12] 2015 & random number & single-blind & unclear & Yes & No & Yes & B \\
\hline Lei Wenlan et al. [13] 2016 & unclear & unclear & unclear & Yes & No & Yes & B \\
\hline Pan Longfang et al. [14] 2015 & random number & single-blind & Yes & Yes & Yes & Yes & A \\
\hline Zhu Ya et al. [15] 2010 & $\begin{array}{l}\text { sequential order of } \\
\text { catheterization }\end{array}$ & unclear & unclear & No & No & Yes & B \\
\hline Lin Cuifang et al. [16] 2015 & random number table & unclear & unclear & No & No & Yes & B \\
\hline Zhang Ronghua et al. [17] 2015 & unclear & unclear & unclear & No & No & Yes & B \\
\hline Zhou Lianqing et al. [18] 2013 & Hospitalization number & unclear & unclear & No & No & Yes & B \\
\hline Zhuo Yajuan et al. [19] 2014 & random number table & unclear & unclear & No & No & Yes & B \\
\hline Xu Mingming et al. [20] 2012 & unclear & unclear & unclear & No & No & Yes & B \\
\hline Jiang Guichun et al. [21] 2016 & envelope method & unclear & unclear & No & No & Yes & B \\
\hline Wang Chaohong et al. [22] 2016 & random number table & unclear & unclear & No & No & Yes & B \\
\hline Li Xiangjun et al. [23] 2016 & unclear & unclear & unclear & No & No & Yes & B \\
\hline Zheng Chunhua et al. [24] 2016 & unclear & unclear & unclear & Yes & No & Yes & B \\
\hline Ren Xiaomin et al. [25] 2016 & random number table & unclear & unclear & No & No & Yes & B \\
\hline
\end{tabular}




\subsection{Meta Analysis Results}

\subsubsection{The Accuracy of Applying Atrial Electro-Gram Technique to PICC Catheter Tip Location}

The accuracy of applying atrial electro-gram technique to PICC catheter tip location was analyzed in 16 randomized controlled studies, and the total sample size was 2996 (there was 1507 cases in atrial electro-gram positioning group, and 1489 cases in X-ray chest positioning group), statistical heterogeneity exists among various studies $\left(\mathrm{RR}=1.13, \mathrm{I}^{2}=79 \%, \mathrm{P}<0.00001\right)$, so the random effect model was used for meta-analysis $(\mathrm{P}<0.05)$. The Meta-analysis showed that the accuracy of the PICC catheter at the lower $1 / 3$ of the superior vena cava in the atrial electro-gram positioning group was higher than that in the X-ray chest positioning group. $[\mathrm{RR}=1.13,95 \% \mathrm{CI}(1.07,1.19), \mathrm{P}<0.00001]$. See Figure 1.

\subsubsection{Effects on the Complications of Applying Atrial Electro-Gram on PICC Catheter Tip Location.}

The results of two studies showed the effect of two different positioning methods on the incidence of complications in PICC catheter. The total number of samples was 643 (323 cases in atrial electro-gram group, 320 cases in X-ray chest group), and there was no heterogeneity among various studies $\left(\mathrm{RR}=0.41, \mathrm{I}^{2}=\right.$ $32 \%, \mathrm{P}<0.21)$. The restorative model was used for meta-analysis. Meta-analysis showed that PICC catheter-related complications in the atrial electro-gram positioning group were lower $(\mathrm{RR}=0.37,95 \% \mathrm{CI}(0.21,0.66), \mathrm{P}<0.0008]$ compared with the X-ray chest positioning group. See Figure 2.

\subsubsection{Effects on Catheterization Time-Consuming of Applying Atrial Electro-Gram on PICC Catheter Tip Location}

Two studies compared the time consumed in two different positioning methods. The total number of samples was 350 (174 cases in atrial electro-gram group, and 176 cases in X-ray chest positioning group), statistical heterogeneity exists between two studies ( $\mathrm{RR}=-12.56, \mathrm{I} 2=99 \%, \mathrm{P}<0.00001)$, and the random effects model was used. Meta-analysis showed that PICC catheterization in atrial electro-gram positioning group took less time than in X-ray chest positioning group $[R R=-12.56,95 \% \mathrm{CI}(-18.56,-6.57), \mathrm{P}<0.0001]$. See Figure 3.

\section{Discussion}

\subsection{The Application of Atrial Electro-Gram to PICC Catheter Tip Location has Improved the Positioning Accuracy.}

This study evaluated the accuracy of atrial electro-gram techniques for PICC catheter tip positioning. The results showed that atrial electro-gram has enhanced the positioning accuracy. The principle of atrial electro-gram (ECG) in PICC catheter tip location was mainly based on the saline and the conductivity of blood, which were regarded as probe electrodes. Changes of $\mathrm{P}$ wave in atrial electro-gram (ECG) was picked up, the location of the catheter tip can be determined from the wave mode and amplitude of $\mathrm{P}$ wave. When the catheter tip was just entering the superior vena cava, the shape and amplitude of the $P$ wave did 


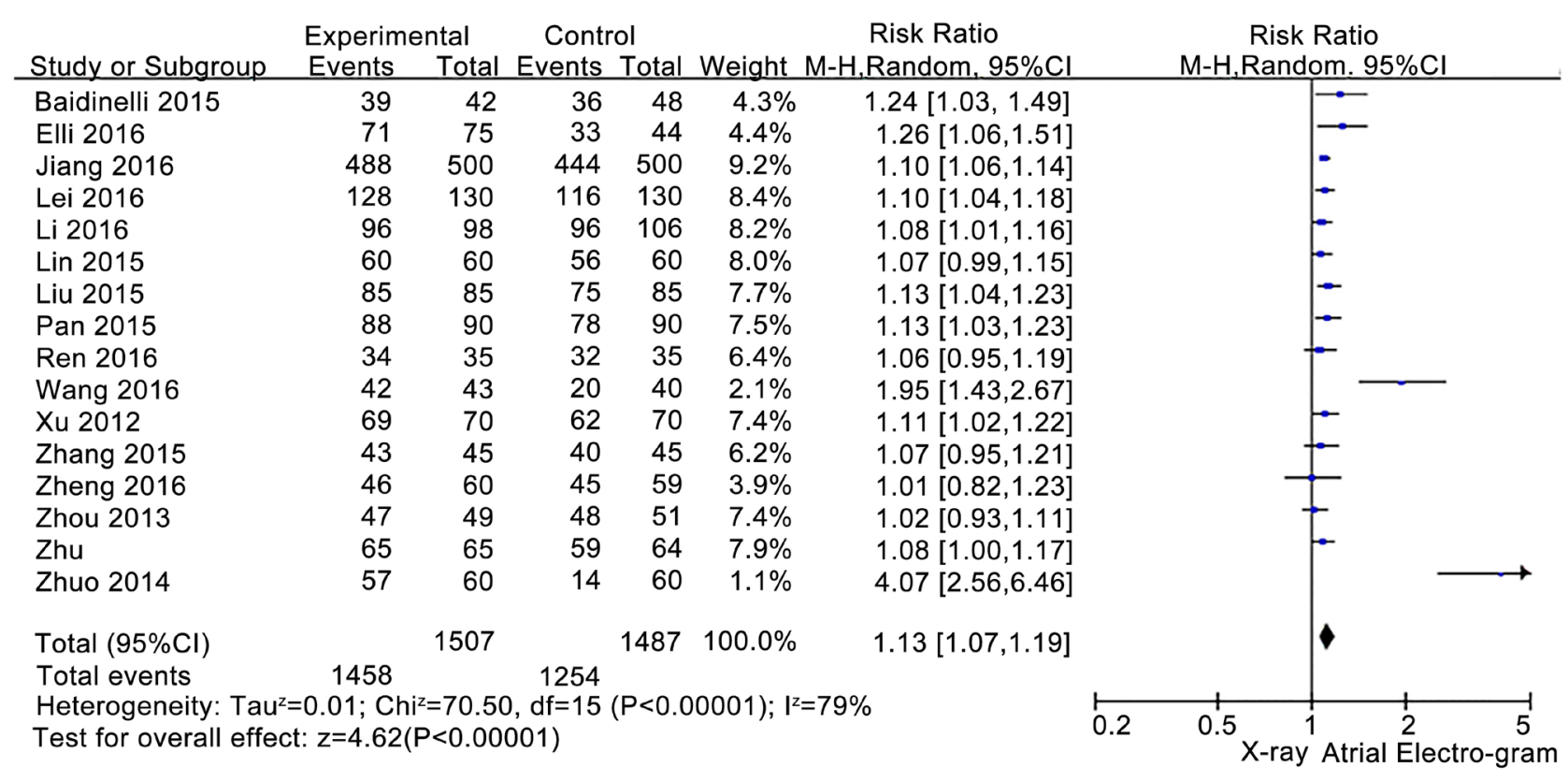

Figure 1. Meta-analysis forest map of applying atrial electro-gram technology in the accuracy of PICC catheter tip positioning.

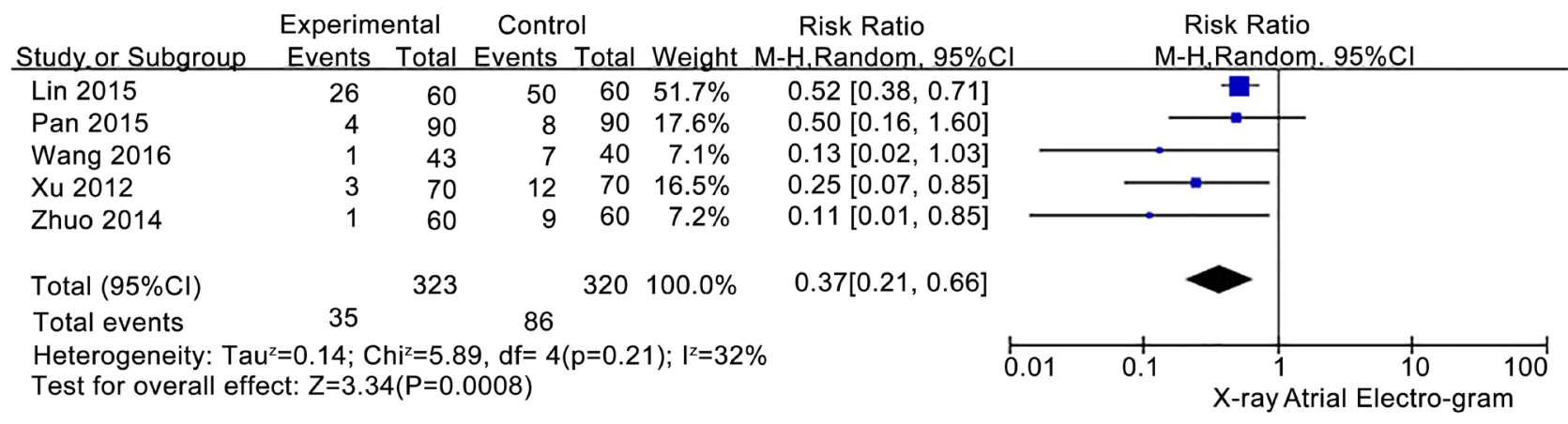

Figure 2. Meta-analysis forest map of applying atrial electro-gram technology in the complications of PICC catheter tip positioning.

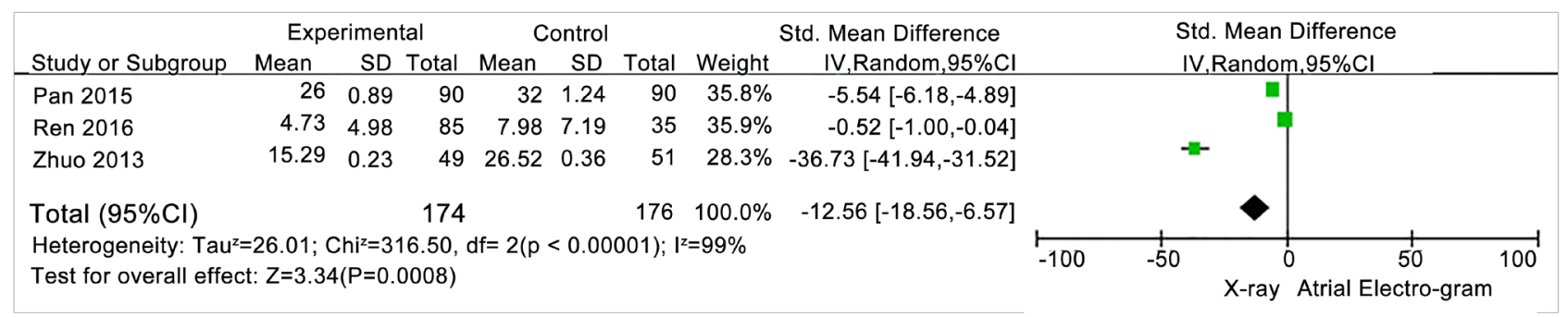

Figure 3. Meta-analysis forest map of applying atrial electro-gram technology in the catheterization time-consuming of PICC catheter tip positioning.

not change; when the catheter was located at the lower $1 / 3$ of the superior vena cava, and about $1-2 \mathrm{~cm}$ from the cavoatrial Junction (CAJ), the upright $\mathrm{P}$ wave with the amplitude equivalent to $50 \%-80 \%$ of the QRS wave was introduced; when the catheter tip was located at the CAJ, the upright $\mathrm{P}$ wave with the amplitude equivalent to QRS waveform was introduced; when the catheter When the 
head end is located in the right atrium, leads to the biphasic $\mathrm{P}$ wave; when the catheter tip was located in the right atrium, diphasic $\mathrm{P}$ wave was introduced; when the catheter tip was located in the ventriculus dexter, negative $\mathrm{P}$ wave that showed double peaks was introduced [26] [27] [28]. During the catheterization process, the nurse can determine the position of the catheter tip according to the change of the $\mathrm{P}$ wave, and timely debug the catheter until a specific $\mathrm{P}$ wave appears. The traditional X-ray chest positioning mainly based on in-vitro prediction length before puncture, which is susceptible to be affected by puncture measurement method, puncture point changes and puncture subjective factors. As a result, the catheter tip can be too deep, too shallow or ectopic. The above situation can't be identified by the naked eye, which can only be determined relying on the chest radiography after the catheterization, so the PICC catheter tip accuracy is greatly reduced. In the early 1990s, atrial electro-gram techniques have been applied to central venous catheters tip positioning in European countries [29]. Over the past decade, many clinical studies have further confirmed that atrial electro-gram location is more accurate than traditional X-ray positioning [30], Gebhard [31] and other studies also show that atrial electro-gram is more secure, more convenient and more reliable than X-ray positioning.

\subsection{The Application of Atrial Electro-Gram to PICC Catheter Tip Location has Reduced the Occurrence Rate of Complications}

Included literature research results showed that the use of atrial electro-gram positioning greatly reduced the incidence of related complications than the traditional X-ray positioning method. Studies have shown that during the PICC catheterization process, it is possible the catheter tip can't be accurately placed in the ideal position as some patients had vascular anatomical changes and other diseases. As a result, the catheter tip needs repeated debugging after ectopia, and the incidence of mechanical vein inflammation, catheter-related bloodstream infection, thrombosis, lymphatic leakage and other complications can be increased [32]. For patients suffered from the chest and ascites diseases, when the catheter is conveyed to the predicted length, the tip may have shifted to the right atrium, and serious complications may be lead to, such as heart rate disorders, heart thrombosis, pericardial tamponade and pericardial rupture that endanger the lives of patients [33]. At present, PICC catheter tip positioning approach used in the clinical combines X-ray film with the estimated length in vitro. After the catheter is conveyed to the predicted length, the guide wire will be removed, and the catheter will be fixed before filming. If the catheter is found to be ectopic, it has to be conveyed again, otherwise, it will be difficult to achieve the ideal position, and the chance of catheter infection or catheter failure will be greatly increased. The application of atrial electro-gram positioning method can always adjust the location of the catheter tip in the aseptic state with $\mathrm{P}$ wave changes, until it is located at the ideal location, which can effectively avoid a number of complications caused by repeated puncture, such as phlebitis, thrombosis, catheter-related bloodstream infection and so on. 


\subsection{The Application of Atrial Electro-Gram to PICC Catheter Tip Location has Reduced the Catheterization Time}

The results of this study (Figure 3) showed that atrial electro-gram positioning method can save the catheterization time compared with the traditional X-ray positioning method. When the X-ray positioning method is used for PICC catheter tip positioning, the location of the tip can only be pre-determined based on the length of in vitro measurement and it is also necessary to go to the radiology department for routine filming. [34] [35]. If the ectopia occurs, you need to re-establish the aseptic area and film again, until the catheter tip location is correct. As we can see this process wastes a lot of valuable time of not only patients but also nurses. Atrial electro-gram positioning method is able to monitor the catheter tip position in real time during the process of catheterization, and adjust any ectopia in time to achieve the integration between catheter and positioning. In addition, it is not necessary for patients to have X-ray examination before the usage of medication, so its advantages are quite prominent [36] for critical patients. In this way, a lot of unnecessary troubles can be saved to ensure the timeliness of treatment. A large sample study carried out by Mourean [6] and other researchers confirmed that the use of atrial electro-gram catheter tip positioning is able to reduce the catheterization time; generally, about $30 \mathrm{mi}$ nutes of operation time can be saved on average, which is consistent with the results of this study.

\subsection{Limitations of the Study}

Since the literature included in this study included only published Chinese and English literature, publication bias may exist for incomplete search; the meta-analysis included 16 randomized controlled trials, and the sample sizes range from 70 to 500 cases, so methodological and sample heterogeneity exists; the quality of the 16 literature included are graded to be lower-intermediate, which is not high. Only one literature [14] uses randomized grouping, strictly follows the blind method, sticks to the allocation concealment and focuses on the loss to follow-up, as well as reports the outcome indicators completely; while the remaining 15 studies all have flaws in the methodology. The detailed grouping methods fail to be described in six studies [10] [13] [17] [20] [23] [24], thus patients in both groups may have no comparability in baseline information and result differences will be large. In addition, Thirteen studies [11] [13] [15]-[25] don't abide by the blind method and it may affect accuracy rate of judging outcome indicators, resulting in mutual pollution of outcome indicators of patients and affecting statistical result differences of patients in both groups. Eleven studies [10] [15]-[23] [25] don't report some information of exit and loss to follow-up, thus patients in both groups may lose some samples with varying degrees and final observational samples may be changed. Consequently, statistical results have no clinical difference. Research data in the study are complete and have no selective report. It is thus clear that seldom ECG in atriums is actually applied in the randomized controlled trial of PICC conductor housing position- 
ing. Meanwhile, literature quality evaluation is intermediate level or below. In the future, it is necessary to popularize and generalize randomized controlled trial, improve clinical researchers' research quality in the randomized controlled trial, and control bias through rigorous experimental design, so as to ensure research quality. It can be seen that there is few randomized controlled trials for atrial electro-gram to be actually conducted applied to PICC catheter tip positioning. In the future, we should strengthen the popularization and promotion of randomized controlled research methods, improve the quality of randomized controlled trials of clinical researchers, and control the bias caused by various reasons through rigorous experimental design, so as to ensure the quality of research. In addition, literatures in the study give no explanations to the positioning security of ECG in atriums and it is short of some evaluations that whether its security will affect final indicators, thus the future study should pay more attention to the positioning security of ECG in atriums. Limited by literature quality and quantity, the study still has some limitations, thus more samples should be used for the randomized controlled trial with the longer duration in the future, so as to further explore and verify superiority and comprehensive effects of positioning PICC conductor housing of ECG in atriums.

In summary, the existing studies show that atrial electro-gram applied to PICC catheter tip positioning can improve the arrival accuracy of catheter tip, reduce the incidence of related complications and save the catheterization time, which can also provide scientific basis for further promotion of the atrial electro-gram positioning method. However, in view of the quality limitations of the literature included in this study, multi-center and large sample of randomized controlled studies are still required in the future to further explore and confirm the superiority of applying atrial electro-gram to PICC catheter tip positioning.

\section{References}

[1] Yao, H. (2012) Clinical Research of Intravenous Electro-Gram on PICC Tip Location. Liaoning Medical College, Jinzhou.

[2] Deng, Z.Y., Chen, M., Huang, M.Y., et al. (2012) Effect Analysis of Intravenous Electro-Gram on PICC Tip Location. Hainan Medical Journal, 23, 116-117.

[3] Oliver, G. and Jones, M. (2014) ECG or X-Ray as the "Gold Standard” for Establishing PICC-Tip Location. British Journal of Nursing, 23, S10-S16. https://doi.org/10.12968/bjon.2014.23.Sup19.S10

[4] Liu, H.M. and Tan, J. (2012) Application of New Catheter Technique in PICC Catheterization Patients Guided by Atrial Electro-Gram. Journal of Qilu Nursing, 18, 43-44.

[5] Li, Q.L., Yan, M.Q., Zhang, X.J., et al. (2013) Study on the Effect of Different PICC Catheters on the Complications. Chinese Journal of Nursing, 48, 390-395.

[6] Mourean, N.L., Dennis, G.L., Ames, E., et al. (2010) Electorcardiogram (EKG) Guided Peripherally Inserted Central Catheter Placement and Tip Position: Results of Trial to Radiological Confirmation. Journal of the Association for Vascular Access, 15, 8-14. https://doi.org/10.2309/java.15-1-3

[7] Hu, J.X., Li, P. and Liu, M.X. (2016) Meta-Analysis on Kangaroo Nursing to Pro- 
mote Neonatal Breastfeeding. Nursing Journal, 23, 9-13.

[8] Kuang, Y.H., Xu, Y.L., Yi, H.L., et.al. (2016) Meta-Analysis of Health Education Intervention on Treatment Compliance of CPAP Patients. Nursing Journal, 23, 13-18.

[9] Hu, Y. (2012) Evidence-Based Nursing. People's Health Publishing House, Beijing, 73-74.

[10] Liu, Y.J., Dong, L., Lou, X.P., et al. (2015) Evaluating ECG-Aided Tip Localization of Peripherally Inserted Central Catheter in Patients with Cancer. International Journal of Clinical and Experimental Medicine, 8, 14127-14129.

[11] Elli, S., Cannizzo, L., Marini, A., et al. (2016) Evaluation of Tip Location Reliability of Intraprocedural ECG vs. Chest X-Rays in PICC Placement. Assistenza Infermieristica $E$ Ricerca, 35, 174-179.

[12] Baldinelli, F., Capozzoli, G., Pedrazzoli, R., et al. (2015) Evaluation of the Correct Position of Peripherally Inserted Central Catheters: Anatomical Landmark vs. Electrocardiographic Technique. The Journal of Vascular Access, 16, 394-398. https://doi.org/10.5301/jva.5000431

[13] Lei, W. and Yan, X. (2016) Application Effect Observation on Central Venous Catheter Tip Positioning under the Guidance of Intraline Electro-Gram. West China Medical, 31, 1-3.

[14] Pan, L., Hong, Y., Tang, L., et al. (2015) Clinical Application Research of Atrial Electro-Gram Positioning of PICC Puncture Technique. Journal of Chongqing Medical University, 40, 472-476.

[15] Zhu, Y., Ge, X., Huang, Y., et al. (2010) Effects of Intravenous Venous Catheter Vein Catheter on Ectopia Cordis by ECG. Nursing Journal of Chinese People's Armed Police Forces, 11, 1711-1712.

[16] Lin, C., Xiao, Y. and Liu, Y. (2015) Effects of Electrocardiogram on PICC Tip Position and Catheterization Complications. Journal of Nursing, 30, 38-40.

[17] Zhang, R., Zhang, J. and Qiu, J. (2015) Clinical Practice and Analysis of ECG Guided PICC Catheter Tip Positioning. Chinese and Foreign Medicine, No. 34, 68 70.

[18] Zhou, L., Chen, Y., Wang, J., et al. (2013) Clinical Application Research of Atrial Electro-Gram Guided PICC Tip Positioning Method. Journal of Nurses Training, 28, 2021-2023.

[19] Zhuo, Y., Feng, L., Jiang, J., et al. (2014) Clinical Application of New Intravenous Electro-Gram Guided PICC Catheter Tip Positioning. Rehabilitation, 13, 868-870.

[20] Xu, M., Wang, T., Yan, J., et al. (2012) Effect Observation of Applying ECG Guidance Method to Improve the Success Rate of PICC Catheterization. Journal of Nursing, 19, 66-67.

[21] Jiang, G. (2016) Effect Evaluation of Applying Atrial Electro-Gram to Assist PICC Catheter Tip Positioning. Chinese Nursing Management, 16, 743-746.

[22] Wang, C., Wang, Z., Hou, X., et al. (2016) Application of Atrial Electro-Gram in PICC Catheterization in Cancer Patients. Medical Equipment, 29, 21-22.

[23] Li, X., Li, L. and Zhang, Y. (2016) Effect Research of Atrial Electro-Gram Positioning on Reducing PICC Catheter Ectopia. Journal of Qiqihar Medical College, 37, 3723-3725.

[24] Zheng, C. and Wu, X. (2016) Clinical Application of Intracardiac Electro-Gram Locates PICC the Catheter Tip Position. Nursing Research, 30, 4208-4209.

[25] Ren, X., Zhang, P., Li, L., et al. (2016) Study on the Positioning of PICC Based on Improved ECG Positioning System. Journal of Nursing Science, 31, 1-4. 
[26] Yao, H., Song, M. and Liu, Y. (2011) Clinical Study of Intravenous Electro-Gram Guided PICC Tip Positioning. Chinese Journal of Nursing, 46, 26-28.

[27] Feng, B., Yao, S., Zhou, S., et al. (2010) Intracardiac Electro-Gram Changes in the Process of Catheterization and the Guidance Role on Catheter Manipulation. Chinese Journal of Nursing, 45, 26-28.

[28] Guo, L., Qin, Y., Wang, G., et al. (2015) The Stability and Influencing Factors of Intracardiac Electro-Gram in Adult Venous Catheter. Chinese Journal of Nursing, 50, 724-727.

[29] Schummer, W., Schummer, C., Schelenz, C., et al. (2004) Central Venous Catheters-The Inability of "Intra-Atrial ECG" to Prove Adequate Positioning. British Journal of Anaesthesia, 93, 193-198. https://doi.org/10.1093/bja/aeh191

[30] Schummer, W., Schummer, C., Schelenz, C., et al. (2005) Modified ECG-Guidance for Optimal Central Venous Catheter Tip Positioning. A Transesophageal Echocardiography Controlled Study. Anaesthesist, 54, 983-990. https://doi.org/10.1007/s00101-005-0886-2

[31] Gebhard, R.E., Szmuk, P., Pivalizza, E.G., et al. (2007) The Accuracy of Electrocardiogram-Controlled Central Line Placement. Anesthesia \& Analgesia, 104, 65-70. https://doi.org/10.1213/01.ane.0000250224.02440.fe

[32] Capozzoli, G., Accinelli, G., Fabbro, L., et al. (2012) Intra-Cavitary ECG Is an Effective Method for Correct Positioning the Tip of Tunneled Groshong Catheters. The Journal of Vascular Access, 13, 393-396. https://doi.org/10.5301/jva.5000065

[33] Walker, G., Chan, R.J., Alexandrou, E., et al. (2015) Effectiveness of Electrocardiographic Guidance in CVAD Tip Placement. British Journal of Nursing, 24, 28-30. https://doi.org/10.12968/bjon.2015.24.sup14.s4

[34] Chen, G., Wang, H. and Zhao, R. (2011) Progress in the Positioning of the Central Venous Catheter by Peripheral Vein Puncture. Chinese Journal of Nursing, 46, 1037-1039.

[35] Peng, X., Wen, Q. and Yao, Q. (2012) Progress on PICC Tip Positioning Method. Nursing Management Journal, 12, 861-862.

[36] Vesely, T.M. (2003) Central Venous Catheter Tip Position: A Continuing Controversy. Journal of Vascular and Interventional Radiology, 14, 527-534. https://doi.org/10.1097/01.RVI.0000071097.76348.72 
Submit or recommend next manuscript to SCIRP and we will provide best service for you:

Accepting pre-submission inquiries through Email, Facebook, LinkedIn, Twitter, etc. A wide selection of journals (inclusive of 9 subjects, more than 200 journals)

Providing 24-hour high-quality service

User-friendly online submission system

Fair and swift peer-review system

Efficient typesetting and proofreading procedure

Display of the result of downloads and visits, as well as the number of cited articles Maximum dissemination of your research work

Submit your manuscript at: http://papersubmission.scirp.org/

Or contact ym@scirp.org 